\title{
Specific oxylipins enhance vertebrate hematopoiesis via the receptor GPR132
}

\author{
Jamie L. Lahvic ${ }^{a, b}$, Michelle Ammerman ${ }^{a, b}$, Pulin Li ${ }^{a, b}$, Megan C. Blair ${ }^{a, b}$, Emma R. Stillman ${ }^{a, b}$, Eva M. Fast ${ }^{c, d}$, \\ Anne L. Robertson ${ }^{a, b}$, Constantina Christodoulou ${ }^{a, b}$, Julie R. Perlin ${ }^{a, b}$, Song Yang ${ }^{a, b}$, Nan Chiang ${ }^{e}$, Paul C. Norris ${ }^{e}$, \\ Madeleine L. Daily ${ }^{a, b}$, Shelby E. Redfield ${ }^{a, b}$, Iris T. Chan ${ }^{a, b}$, Mona Chatrizeh ${ }^{a, b}$, Michael E. Chase ${ }^{a, b}$, Olivia Weis ${ }^{a, b}$, \\ Yi Zhou ${ }^{a, b}$, Charles N. Serhan ${ }^{\mathrm{e}}$, and Leonard I. Zon ${ }^{\mathrm{a}, \mathrm{b}, \mathrm{c}, \mathrm{d}, \mathrm{f}, 1}$
}

aStem Cell Program, Boston Children's Hospital, Boston, MA 02115; 'Division of Hematology/Oncology, Boston Children's Hospital, Boston, MA 02115;
'Harvard Stem Cell Institute, Harvard University, Cambridge, MA 02138; 'Department of Stem Cell and Regenerative Biology, Harvard University,
Cambridge, MA 02138; 'Center for Experimental Therapeutics and Reperfusion Injury, Department of Anesthesiology, Perioperative and Pain Medicine,
Brigham and Women's Hospital, Boston, MA 02115; and 'Howard Hughes Medical Institute, Boston Children's Hospital, Boston, MA 02115

Edited by Gregg L. Semenza, Johns Hopkins University School of Medicine, Baltimore, MD, and approved July 30,2018 (received for review April 11, 2018)

Epoxyeicosatrienoic acids (EETs) are lipid-derived signaling molecules with cardioprotective and vasodilatory actions. We recently showed that 11,12-EET enhances hematopoietic induction and engraftment in mice and zebrafish. EETs are known to signal via G protein-coupled receptors, with evidence supporting the existence of a specific high-affinity receptor. Identification of a hematopoieticspecific EET receptor would enable genetic interrogation of EET signaling pathways, and perhaps clinical use of this molecule. We developed a bioinformatic approach to identify an EET receptor based on the expression of $\mathrm{G}$ protein-coupled receptors in cell lines with differential responses to EETs. We found 10 candidate EET receptors that are expressed in three EET-responsive cell lines, but not expressed in an EET-unresponsive line. Of these, only recombinant GPR132 showed EET-responsiveness in vitro, using a luminescencebased $\beta$-arrestin recruitment assay. Knockdown of zebrafish gpr132b prevented EET-induced hematopoiesis, and marrow from GPR132 knockout mice showed decreased long-term engraftment capability. In contrast to high-affinity EET receptors, GPR132 is reported to respond to additional hydroxy-fatty acids in vitro, and we found that these same hydroxy-fatty acids enhance hematopoiesis in the zebrafish. We conducted structure-activity relationship analyses using both cell culture and zebrafish assays on diverse medium-chain fatty acids. Certain oxygenated, unsaturated free fatty acids showed high activation of GPR132, whereas unoxygenated or saturated fatty acids had lower activity. Absence of the carbon-1 position carboxylic acid prevented activity, suggesting that this moiety is required for receptor activation. GPR132 responds to a select panel of oxygenated polyunsaturated fatty acids to enhance both embryonic and adult hematopoiesis.

hematopoiesis | free fatty acid | GPCR | zebrafish

$E^{\mathrm{i} i}$ cosanoids are endogenous bioactive mediators derived from arachidonic acid, and are responsible for a variety of physiological phenotypes (1). Epoxyeicosatrienoic acids (EETs), a major class of eicosanoids formed by cytochrome P450 enzymes, play critical roles in endothelial migration, monocyte adhesion, tumor metastasis, and vasodilation, among other cell-typespecific effects (2-4). We recently showed that 11,12-EET enhances the specification of hematopoietic stem and progenitor cells (HSPCs) in developing zebrafish embryos, as well as the transplant of HSPCs in both fish and mice (5). Despite the important physiological roles of EETs, their direct protein target(s) remain unknown.

Previous results indicate that EETs bind to at least one specific G protein-coupled receptor (GPCR). Nanomolar or even picomolar concentrations of 11,12- or 14,15-EET can elicit specific cellular phenotypes $(6,7)$, and a bead-tethered EET without the ability to cross the plasma membrane maintained its activity (8), suggesting the existence of a high-affinity, membrane-bound EET receptor. Chen et al. (9) demonstrated that U937 and other EET-responsive cell lines express a single high-affinity EET receptor of about $47 \mathrm{kDa}$ in size. This is likely a GPCR, as EETs require $\mathrm{G}$ protein signaling components to elicit many phenotypes (10-12). For instance, we previously showed that EET's enhancement of zebrafish hematopoiesis requires signaling via $\mathrm{G} \alpha 12 / 13$ (5).

As traditional biochemical methods have so far failed to identify EET receptors, here we used bioinformatic techniques to identify candidate receptors and assayed those candidates for EET responsiveness in vitro. Only GPR132 (G2A), a previously described fatty acid receptor (13-16), showed responsiveness to EET. We demonstrated that GPR132 is required for EETinduced hematopoietic stem cell specification in the zebrafish and for normal hematopoietic stem cell transplant in the mouse. Previously described fatty acid activators of GPR132 induced hematopoietic phenotypes in the zebrafish essentially identical to those observed with EET, further confirming that these molecules activate the same pathway. We performed structure-activity relationship analyses to determine the full range of GPR132 activators. Rather than a regiospecific, high-affinity receptor, our data show that GPR132 is likely a low-affinity, promiscuous receptor for a select panel of oxygenated polyunsaturated fatty acids, whose activity drives hematopoiesis in embryonic and adult vertebrate contexts.

\section{Significance}

Small-molecule enhancers of hematopoietic stem cell transplant could improve the safety of this treatment and expand the pool of eligible patients. We previously showed that the lipid 11,12-epoxyeicosatrienoic acid (EET) enhanced transplant in zebrafish and mice. We use a bioinformatic approach to identify candidate EET receptors and demonstrate that EET activates GPR132. We find that this receptor is important in zebrafish and mouse hematopoiesis, and we further show that GPR132 has responsiveness to additional oxygenated polyunsaturated fatty acids such as EET. Thus, GPR132 receives lipid-derived signals to regulate hematopoiesis and is a therapeutic target for enhancing HSC transplant.

Author contributions: J.L.L., P.L., J.R.P., N.C., Y.Z., C.N.S., and L.I.Z. designed research; J.L.L., M.A., P.L., M.C.B., E.R.S., E.M.F., A.L.R., C.C., J.R.P., N.C., P.C.N., M.L.D., S.E.R., I.T.C., M.C., M.E.C., and O.W. performed research; J.L.L., E.M.F., C.C., S.Y., C.N.S., and L.I.Z. analyzed data; and J.L.L., P.L., E.M.F., C.N.S., and L.I.Z. wrote the paper.

The authors declare no conflict of interest.

This article is a PNAS Direct Submission.

Published under the PNAS license.

Data deposition: The data reported in this paper have been deposited in the Gene Ex pression Omnibus (GEO) database, https://www.ncbi.nlm.nih.gov/geo (accession no. GSE113550).

${ }^{1}$ To whom correspondence should be addressed. Email: zon@enders.tch.harvard.edu.

This article contains supporting information online at www.pnas.org/lookup/suppl/doi:10 1073/pnas.1806077115/-/DCSupplemental.

Published online August 23, 2018. 


\section{Results}

Identification of Candidate EET Receptors. EETs elicit phenotypes only in specific cell types, suggesting that a putative EET receptor might be selectively expressed in these cell types. We performed RNAseq profiling in duplicate on three previously identified EET-responsive human cell lines [U937 monocytes (17), EaHy endothelial cells (18), and PC3M-LN4 prostate cancer cells (4)], two of which show binding to a radiolabeled EET analog (9). We profiled a fourth cell line (HEK293) that has no known responsiveness to EET and shows no such binding (9). Although we detected reads for hundreds of GPCRs (SI Appendix, Fig. S1), only 37 GPCRs were expressed in common in all three EET-responsive cell lines above 0.3 fragments per kilobase per million reads (FPKM) (SI Appendix, Fig. S2A), a conservative threshold for physiologically meaningful abundance. Of these, 27 were also expressed at moderate to high levels $(\mathrm{FPKM}>0.9)$ in our EET nonbinding cell line (SI Appendix, Fig. S2B). This left 10 candidate EET receptors that were expressed only in EET binding cell lines and were missing from the nonbinding cell line. All candidates had predicted molecular weights within $20 \%$ of the predicted $47-\mathrm{kDa}$ size of an EET receptor (9), and candidates included both wellstudied GPCRs such as the $\beta$-adrenergic receptor and prostaglandin receptors and orphan GPCRs such as GPR132 and GPR135 (SI Appendix, Fig. S2C).

EET Activates $\beta$-Arrestin Recruitment via GPR132 in Vitro. GPCR activation causes recruitment of $\beta$-arrestin, which can be measured with the luminescence-based PathHunter assay (13, 15, $19)$. We tested for EET-induced $\beta$-arrestin recruitment via each candidate GPCR with the exception of GPR68, PTGER1, and LPAR6, which have no available PathHunter assays (Fig. 1). Although candidates with known cognate small molecule ligands showed robust and dose-dependent activation by those ligands (Fig. $1 C-F$ ), EET showed no activity in these assays. CCRL2, a receptor for specific peptides, and GPR135, an orphan receptor, also failed to respond to EET (Fig. $1 G$ and $H$ ). In contrast, 11,12-EET dose-dependently recruited $\beta$-arrestin to the GPR132 receptor, also known as $\mathrm{G} 2 \mathrm{~A}$, in an apparently low-affinity interaction (Fig. $1 B$ ). Interestingly, two groups have previously assayed GPR132's ability to respond to 14,15-EET and failed to see a high-affinity response $(9,20)$. We found GPR132 to be more responsive to 11,12-EET than 14,15-EET (Fig. 1B). GPR132 is a member of the GPR4 family of GPCRs, which are hypothesized to be lipid-sensing or acid-sensing receptors (21-24). In addition to GPR132, this family includes GPR4, GPR65, and GPR68, an additional candidate from our bioinformatic analysis. No $\beta$-arrestin assay is available for GPR68, but 11,12-EET showed no activation of GPR4 or GPR65 (SI Appendix, Fig. S3). Together, these results suggest 11,12-EET specifically binds to and activates GPR132, although this interaction may not be high affinity.

gpr132b Is Required for EET-Induced Enhancement of Zebrafish Hematopoiesis. To determine whether GPR132 is required for EET phenotypes, we investigated EET-induced hematopoietic phenotypes in zebrafish embryos. We previously showed that EET treatment from 24 to $36 \mathrm{~h}$ postfertilization (hpf) enhances specification of HSPCs in the aorta-gonad-mesonephros (AGM) region (5). Later EET treatment enhances homing of HSPCs to the caudal hematopoietic territory (CHT), a developmental niche akin to the human fetal liver (5). Zebrafish have two GPR132 homologs, gpr132a and gpr132b. At the amino acid level, they share $39 \%$ and $44 \%$ sequence similarity with human GPR132, respectively (22). Previous RT-PCR studies showed gpr132b, but not gpr132a, expression in whole zebrafish embryos (25). We were unable to expand a gpr132a in situ probe from embryonic RNA, perhaps because of low expression of this homolog. To determine where $g p r 132 b$ is endogenously expressed in zebrafish embryos, we performed in situ hybridization (ISH) in wild-type, untreated embryos at 24, 36, and 48 hpf. gpr132b showed expression in the brain, including cells in the anterior lateral line nerve at all stages tested (SI Appendix, Fig. S4). Staining was also seen in the posterior
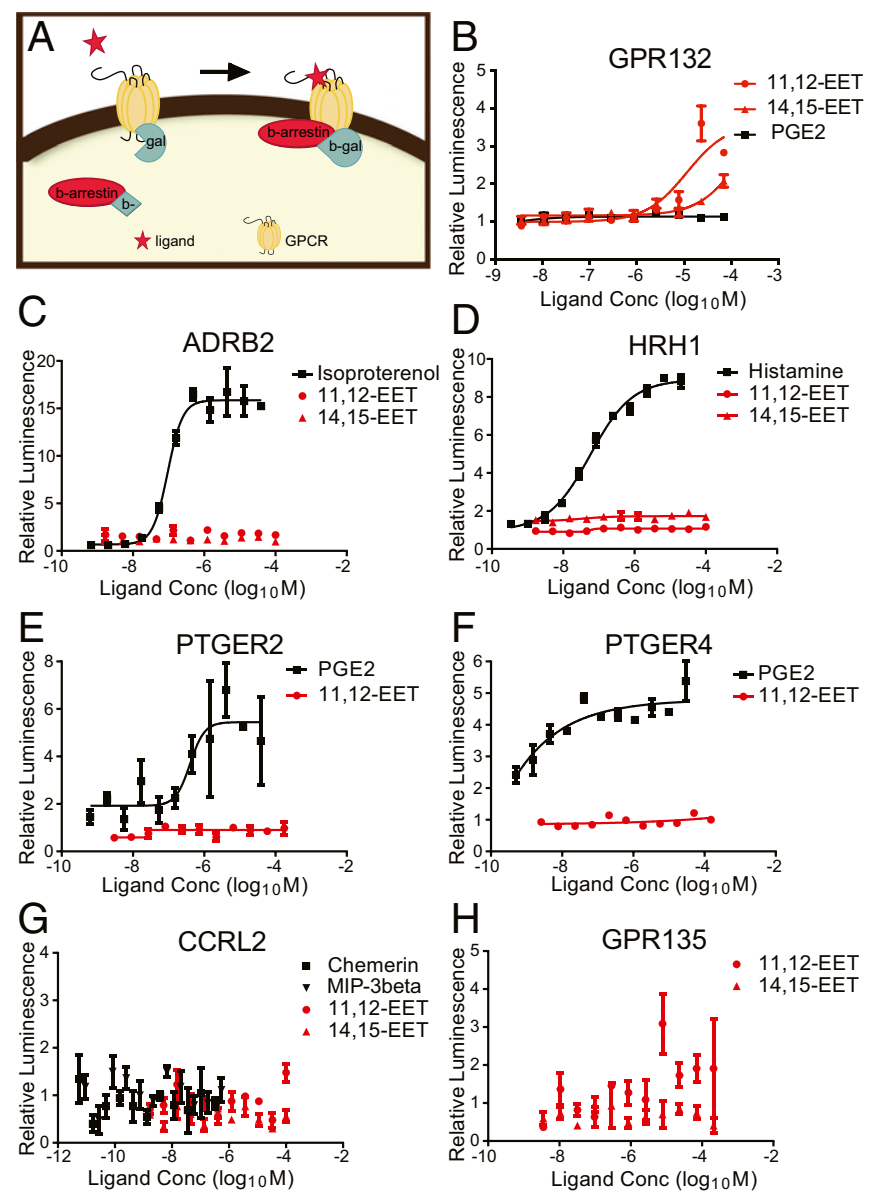

Fig. 1. 11,12-EET activates GPR132 in vitro. (A) Diagram depicting the DiscoverX PathHunter $\beta$-arrestin recruitment assay. Ligand-GPCR binding induces $\beta$-arrestin recruitment, causing complementation of a split- $\beta$-galactosidase enzyme. $(B-H) \beta$-arrestin assays for candidate GPCRs treated with their known ligands or 11,12 - or $14,15-E E T$. PGE2, prostaglandin E2.

spinal cord at $24 \mathrm{hpf}$ (arrows). Although gpr132b expression was not detectable by ISH in the AGM, it is expressed in the CHT. The CHT showed punctate expression (arrowheads) at all three stages, with strongest expression at $36 \mathrm{hpf}$. This correlates with early stages of CHT colonization by myeloid precursors and HSPCs.

We designed a splice-blocking morpholino (MO) against gpr132b and injected 4-6 ng into 1-cell zebrafish embryos. Embryos were then treated from 24 to $36 \mathrm{hpf}$ with $5 \mu \mathrm{M}$ 11,12-EET and fixed at $36 \mathrm{hpf}$ to stain for runx1, a marker for HSPCs. Consistent with previous results, in uninjected or control MOinjected embryos, EET caused an increase in runxl staining in the AGM and induced ectopic runxl staining in the tail mesoderm (Fig. 2) (5). Injection of gpr132b MO completely blocked EET-up-regulated runxl expression in the AGM and partially blocked this up-regulation in the tail (Fig. 2). This difference may reflect differing threshold requirements of gpr132b activity, with a higher level of $g p r 132 b$ required in the AGM than the tail. Alternatively, the second zebrafish ortholog gpr132a may be able to compensate for the loss of gpr132b in the tail, but not the AGM, or low-affinity interactions with additional GPCRs may contribute to the tail phenotype. Coinjections of human GPR132 RNA with the gpr132b MO restored the high AGM runx1 staining on EET treatment, suggesting this MO effect was specific to loss of gpr132b (SI Appendix, Fig. S5). GPR132 is thus required for EET's enhancement of HSPC specification in zebrafish embryos. 
A

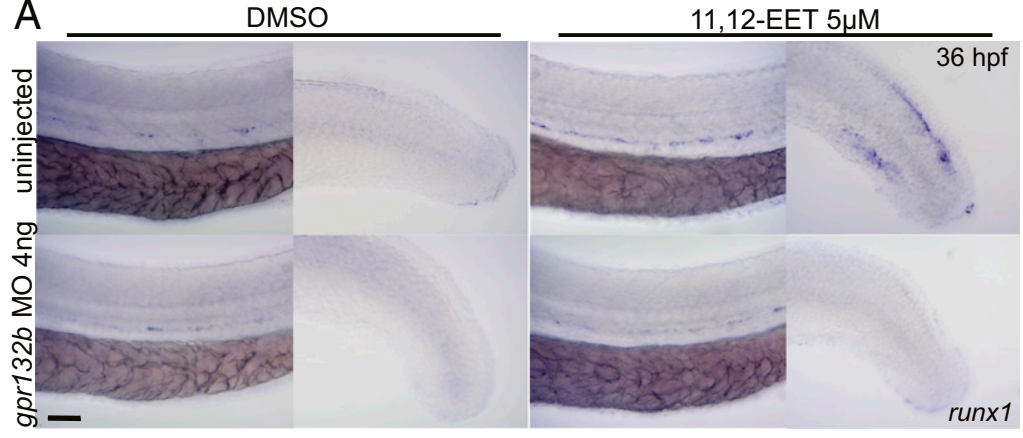

B

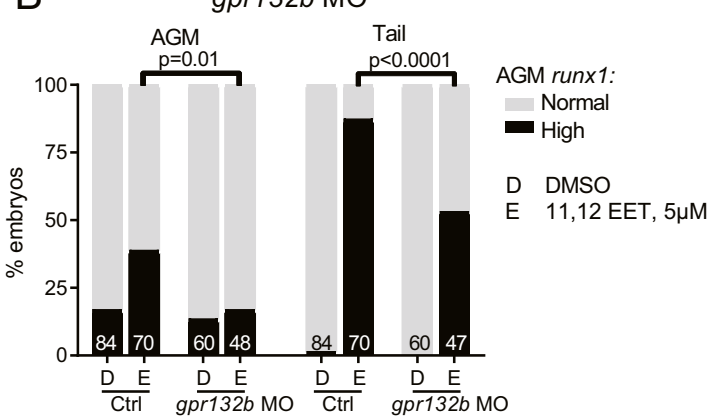

Fig. 2. gpr132b is required for EET's enhancement of zebrafish developmental hematopoiesis. (A) Single-cell zebrafish embryos were injected with 4-6 ng gpr132b MO or control MO or were uninjected, and then treated with DMSO or $5 \mu \mathrm{M} 11,12$-EET beginning at 24 hpf. Embryos were fixed at 36 hpf and stained for runx1 expression. (Scale bar, $100 \mu \mathrm{m}$.) (B) Embryos were scored as having high or normal runx1 expression in the AGM, and high (present) or normal (absent) runx1 expression in the tail mesoderm. Graph presents summary of several experiments, total number of embryos at base of each column. Two-tailed Fisher's exact test. Ctrl includes control MO injected and uninjected embryos.

GPR132 Knockout Mice Show Impaired Long-Term HSC Transplant. GPR132 knockout mice have normal overall physiology, although they are known to show an age-related autoimmune disease featuring an increase in B cells and T cells, as well as lymphocytic infiltration of tissues (26). We found that 3.5-5-moold GPR132 knockout mice have normal distributions of B cells, $\mathrm{T}$ cells, and granulocytes, as well as comparable numbers of phenotypic long-term HSCs, short-term HSCs, and multipotent progenitors compared with their wild-type or heterozygous siblings (SI Appendix, Fig. S6). To stringently test HSPC function, we performed limiting dilution whole-marrow transplants from GPR132 knockout and heterozygous mice. We transplanted donor cells from CD45.1 $1^{+}$, GPR132-/- , or GPR132 $2^{+/-}$mice together with wild-type CD $45.2^{+}$competitor cells into irradiated CD $45.2^{+}$recipients and observed peripheral blood chimerism over time (Fig. 3). At a limiting, 10,000-cell dose, GPR132 $2^{-/-}$ marrow shows a functional defect in competitive long-term engraftment compared with GPR $132^{+/-}$marrow, indicating that GPR132 is required for normal HSC function. This defect was consistent in all blood lineages (SI Appendix, Fig. S7 $A$ and $B$ ) and in the marrow (SI Appendix, Fig. S7C), and resulted in a significantly smaller proportion of knockout mice displaying multilineage chimerism (SI Appendix, Fig. S7D). No difference was seen between heterozygous and knockout marrow at the higher cell dose (Fig. 3B). There was no difference in lineage contribution between heterozygous or knockout marrow at either cell dose (SI Appendix, Fig. S7E). We chose representative recipient mice from each cell dose and used marrow from these to perform secondary transplants (Fig. 3 and SI Appendix, Fig. S7). In general, peripheral blood and marrow chimerism correlated between primary and secondary recipients (SI Appendix, Fig. S8), and knockout marrow continued to show impaired engraftment at the limiting, 10,000-cell dose (Fig. 3 and SI Appendix, Fig. S7 $A$ and $B$ ). Overall, these transplant data suggest that GPR132 knockout mice have a decreased number of functional long-term HSCs compared with their heterozygous siblings, which demonstrates that GPR132 plays an important role in regenerative processes even in the absence of exogenous EET or other small molecule stimulation. The question of how GPR132 signals endogenously in mammalian hematopoietic niches, which could be constitutive or in response to endogenous ligands, is an important area for further study.

Known GPR132 Agonists Activate Hematopoiesis in Developing Zebrafish. GPR132 is a reported receptor for 9-hydroxyoctadecadienoic acid (9-HODE) and 11-hydroxyeicosatetraenoic acid (11-HETE) (13-16). Similar to 11,12-EET, these molecules are oxygenated, polyunsaturated free fatty acids (SI Appendix, Table S1). We confirmed these previous data and showed that both recruited $\beta$-arrestin downstream of GPR132 in vitro dose-dependently (Fig. 4A). In addition, we treated zebrafish embryos with 9-HODE and 11-HETE from 24 to $36 \mathrm{hpf}$ and examined expression of the hematopoietic markers runx1 and $c-m y b$. 9-HODE and 11-HETE produced EETlike phenotypes in zebrafish embryos, increasing runxl/c-myb expression in both the AGM and tail (Fig. 5 and SI Appendix, Table $\mathrm{S} 1$ ). The tail staining of runxl/c-myb is a phenotype we have uniquely seen with EET treatment, despite conducting a wide variety of chemical treatments of embryos with other bioactive lipids such as prostaglandin E2 in our laboratory. The high similarity among EET, 9-HODE, and 11-HETE phenotypes strongly suggests these molecules are operating through the same pathway and activating the same receptor, namely, GPR132.

GPR132 Is Responsive to a Range of Oxygenated Fatty Acids. As GPR132 showed comparable reactivity to 11,12-EET, 9-HODE, and 11-HETE, we sought to further explore potential GPR132 ligands by conducting structure-activity relationship analyses. We assayed a variety of bioactive lipids for their ability to recruit $\beta$-arrestin to GPR132 in vitro and to enhance runx1/c-myb expression in zebrafish embryos in vivo. We found that simple chain, oxygenated, unsaturated free fatty acids including 11,12EET, 11-HETE, 9-HODE, and 11,12-dihydroxyeicosatrienoic acid (11,12-DHET) robustly enhanced both of these phenotypes (Figs. $4 A$ and 5 and SI Appendix, Table S1). More saturated lipids such as 9,10- and 12,13-epoxyoctadecenoic acid (EpOME) showed lower affinity in $\beta$-arrestin assays (Fig. $4 B$ ) and showed little activity in the zebrafish in vivo (Fig. $5 B$ and SI Appendix, Table S1). Fatty acid-derived products such as 13-HODE and 20HETE, with their oxygenated groups displaced farther from the carboxylic acid, similarly showed decreased activity in vitro (Fig. $4 C$ ) and in vivo (SI Appendix, Table S1). Oxygenation of specific polyunsaturated fatty acids appears to contribute to GPR132 activation, as the unoxygenated free fatty acids linoleic acid, eicosapentaenoic acid (EPA), docosahexaenoic acid (DHA), and the unoxygenated EET precursor arachidonic acid showed very little activity in vivo (Fig. $5 B$ and SI Appendix, Table S1). In vitro, these molecules also showed reduced efficacy compared with 11,12-EET (Fig. $4 D$ and $E$ ), with the exception of linoleic acid, which behaved equivalently to 11,12-EET in the $\beta$-arrestin assay (Fig. 4C). 11,12-EET-methyl ester, which replaces the carboxylic acid of 11,12-EET with a methyl group, had no activity either in $\beta$-arrestin assays or in zebrafish embryos, suggesting the carboxylic acid is absolutely required for binding to GPR132 (Figs. $4 E$ and 5 and SI Appendix, Table S1).

11,12-DHET, which showed strong activity in our in vitro and in vivo assays, is a metabolite of 11,12-EET formed rapidly in vivo by the action of soluble epoxide hydrolase ( $\mathrm{sEH})$. To determine whether the actions of 11,12-EET should be attributed to the action of this metabolite, we performed cotreatments of zebrafish embryos with 11,12-EET and two sEH inhibitors, 

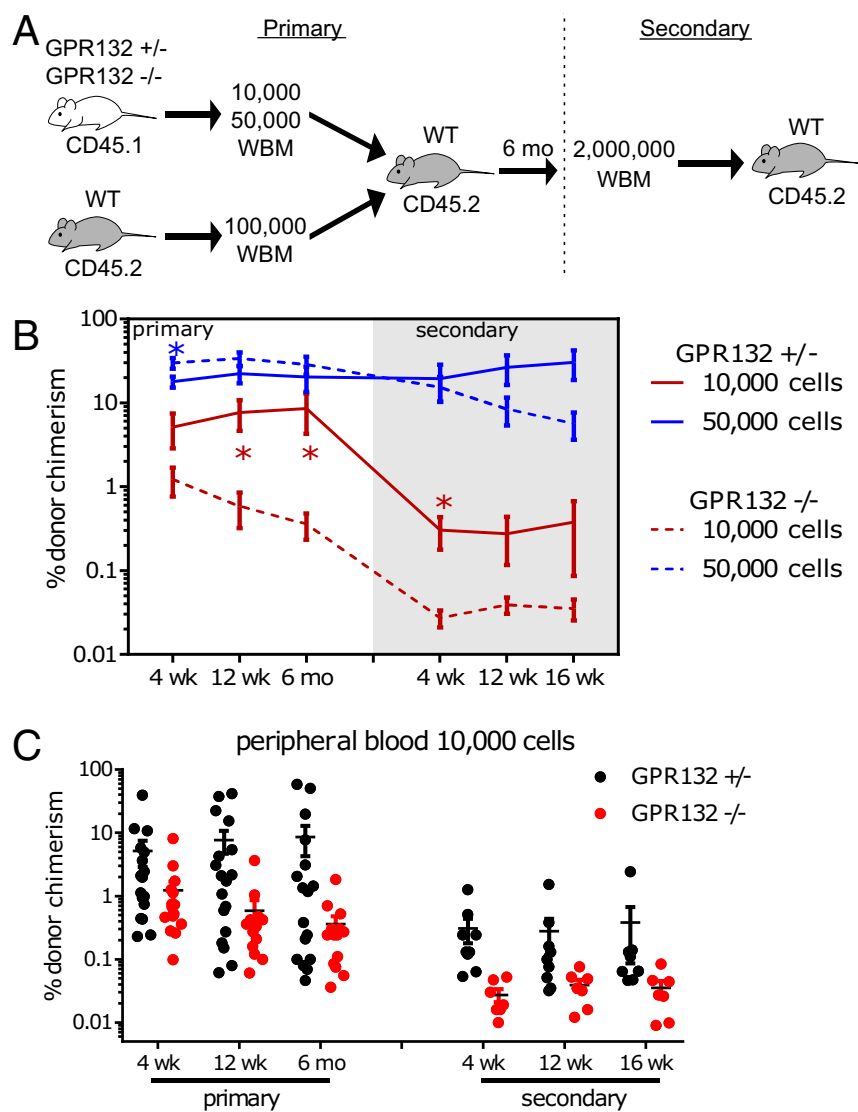

Fig. 3. GPR132 is required for normal marrow transplant in the mouse. $(A)$ 10,000-50,000 donor whole bone marrow (WBM) cells were combined with 100,000 wild-type competitor cells and transplanted into lethally irradiated recipients. Mice were bled at indicated points, and cells were stained for donor chimerism. At 6 mo posttransplant, representative primary recipients were euthanized and 2 million WBM were transplanted into secondary recipients. (B) Peripheral blood chimerism in transplant recipients. Graph summarizes data from three separate experiments with a total of 14-17 primary recipients per condition. Three to six primary recipients were used as donors for three secondary recipients each. Mean with SEM is plotted. ${ }^{*} P<$ 0.05 , Student's $t$ test. (C) Peripheral blood contribution of 10,000 donor cells over time for each genotype in primary and secondary transplant, individual mice shown. Mean with SEM is plotted.

tAUCB and TPPU (27). TPPU had no effect on EET phenotypes, whereas tAUCB decreased EET's enhancement of tail, but not AGM expression of runxl/c-myb (SI Appendix, Fig. S9). The differences between these two drugs may reflect a difference in activity at the zebrafish sEH; only tAUCB has been previously reported to be active in fish (28). The difference seen between tAUCB's effects in the AGM and tail may suggest that DHET is a more potent activator than EET in the zebrafish tail, whereas the two molecules are equally potent in the AGM. Alternatively, tAUCB may be more accessible in the zebrafish tail, as this is a very thin tissue.

Previous studies have hypothesized that GPR132 and its close family members function as acid-sensing receptors (21-24, 29 ), or as receptors for lysophosphatidylcholine (LPC) and sphingosylphosphorylcholine (SPC; reviewed in ref. 30). These results have been controversial, and direct binding experiments linking LPC to GPR132 were later retracted $(31,32)$. We therefore assayed the abilities of these molecules to activate GPR132. In our hands, lactic acid, LPC, and SPC all failed to induce $\beta$-arrestin recruitment by GPR132 (Fig. $4 B$ and SI Appendix, Fig. S10) and failed to enhance HSPC marker staining in zebrafish (Fig. 5B and SI Appendix, Fig. S10). Three additional organic acids also did not cause recruitment of $\beta$-arrestin by GPR132 (Fig. $4 F$ ). Collectively, our data point to GPR132 as a receptor for mediumchain, oxygenated free fatty acids, rather than a broadly $\mathrm{pH}-$ sensitive receptor or an LPC/SPC-responsive receptor.

\section{Discussion}

We employed a bioinformatic pipeline and experiments in zebrafish, a genetically and chemically tractable model organism, to address the long-standing question of the identity of the EET receptor. We report that 11,12-EET enhances HSPC specification in zebrafish embryos by activating the receptor GPR132. This receptor is responsive to a select panel of oxygenated polyunsaturated fatty acids, suggesting that it can integrate these diverse signals in vivo to regulate hematopoiesis. In addition, GPR132 appears to be a conserved regulator of HSPC function in mammals, where loss of this receptor leads to impaired longterm reconstitution after primary and secondary transplant.

In contrast to our results, classically studied EET phenotypes often display high affinity and high regiospecificity (reviewed in ref. 2). For instance, EET stimulation of $\mathrm{BK}(\mathrm{Ca})$ channels is a high-affinity response (7) that is specific to EET and not its metabolite DHET (33). Although certain EET phenotypes appear to be common to both the 11,12-EET and 14,15-EET regioisomers [i.e., both were shown to activate sphingosine kinase activity in human endothelial cells in culture (34)], significant research shows differences in activity and metabolism between the two. SEH shows a strong substrate preference for 14,15-EET over 11,12-EET (35), whereas cyclooxygenases were recently shown to metabolize 11,12-EET, but not 14,15-EET (36). 14,15-EET is a stronger inhibitor of a calcium-regulated potassium channel than 11,12-EET (37), and a stronger activator of free fatty acid receptor 1 (FFAR1, also called GPR40) (38). 11,12-EET, but not other regioisomers, caused changes in the electrical potential of rat tracheal epithelial cells (39) and enhanced endothelial cell migration and tube formation (11). Of interest, in our $\beta$-arrestin assays and zebrafish embryo treatments, 11,12-EET, 14,15-EET, and 11,12-DHET were all active, along with additional oxygenated, polyunsaturated fatty acids. It is possible that the zebrafish GPR132

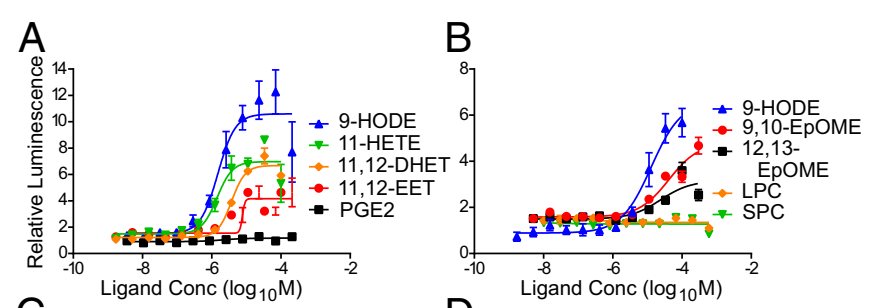

C

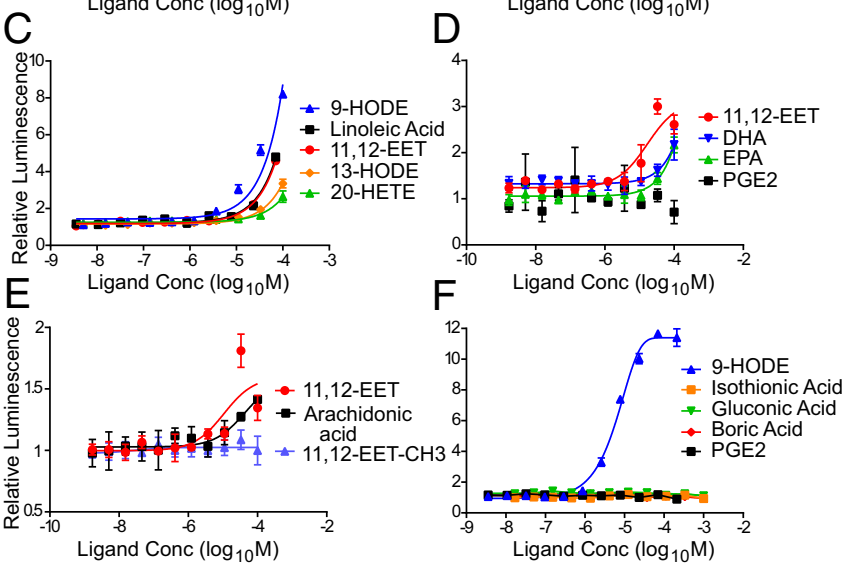

Fig. 4. Diverse free fatty acids activate GPR132 signaling in vitro. (A-F) GPR132 $\beta$-arrestin recruitment assays. Small molecules were treated in triplicate, and each graph represents at least two similar experiments. Error bars represent SEM. Note differing $y$-axis scales. 


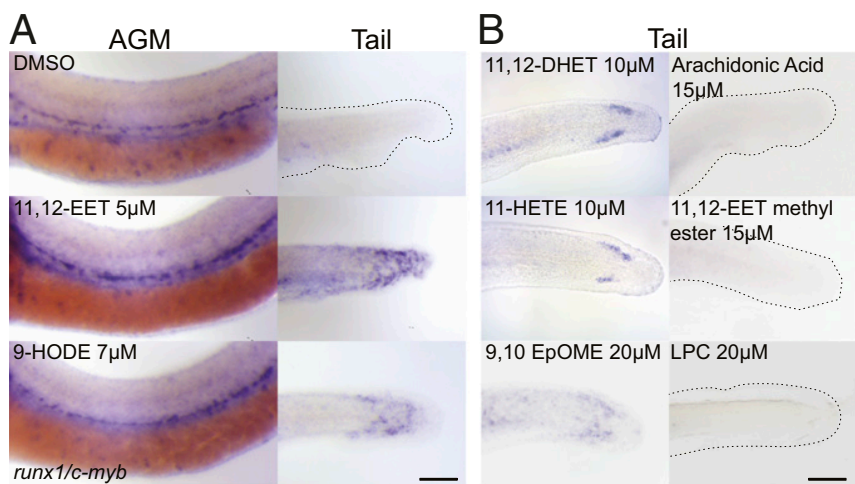

Fig. 5. Fatty acid GPR132 activators enhance expression of HSPC markers in zebrafish embryos. (A) ISH of zebrafish embryos treated with DMSO, 11, 12 EET, or 9-HODE showing AGM and tail expression of runx1/c-myb. For full quantification, see SI Appendix, Table S1. (B) ISH of zebrafish embryos treated with diverse free fatty acids, EET-methyl ester, and LPC showing tail expression of runx1/c-myb. For full quantification, see SI Appendix, Table S1. (Scale bar, $100 \mu \mathrm{m}$. )

is less selective than mammalian receptors for specific lipid mediators. Indeed, 11,12- and 14,15-EET, as well as 11,12-DHET, all showed similar potencies in zebrafish embryo experiments (SI Appendix, Table S1), whereas the $\beta$-arrestin assay for human GPR132 showed a clear preference for 11,12-DHET (Fig. 4A).

Accurate affinity measurements cannot be made from either of our methods because of tissue accessibility challenges in the zebrafish and serum interference with binding in $\beta$-arrestin assays (40). Direct biochemical interrogation of EET-GPR132 binding is still needed. We attempted to assay the ability of 11,12-EET to compete with a tritium-labeled 9-HODE (custom synthesis with American Radiolabeled Chemicals) for potential binding to GPR132. However, we were only able to obtain $\mathrm{H}^{3}-9-\mathrm{HODE}$ with a very low specific activity $(0.13 \mathrm{Ci} / \mathrm{mMol})$ after HPLC isolation and purification, which impeded accurate assessments of specific binding. Other proposed EET receptors include TRPV/TRPC receptors (41-43), peroxisome-proliferatoractivated receptors (44), prostaglandin receptors $(20,45)$, and FFAR1 $(38,46)$. FFAR1 had very low expression in our EaHy samples (average FPKM $=0.14$ ). We tested two prostaglandin receptors and did not obtain responses to EET in vitro (Fig. $1 G$ and $H$ ). Voltage gated channels or nuclear hormone receptors do not explain the $\mathrm{G}$ protein dependence of certain EET phenotypes. Interestingly, however, 9-HODE and 13-HODE are also reported to activate TRPV1 (47). Our results point to GPR132 as a potentially low-affinity EET and oxy-fatty acid receptor with physiological relevance in hematopoiesis. We cannot rule out the existence of additional EET receptors, responsible for other EET phenotypes, which may have high-affinity, regiospecific interactions.

GPR132 itself is a controversial molecule that has been described variously as a receptor for free fatty acids (13-15), a receptor for LPC and SPC, or a proton and $\mathrm{pH}$-sensitive receptor (reviewed in refs. 48 and 49). We saw no evidence of LPC-, SPC-, or $\mathrm{pH}$-responsiveness of GPR132. Our data instead support the role of GPR132 as a free fatty acid receptor and further define that maximum activity is achieved with medium chain, multiply unsaturated, oxygenated fatty acids. Heterodimerization of GPR132 with other GPCRs could explain the differing ligand sensitivities seen by many groups. One overexpression study suggested that GPR132 heterodimerizes with its close family member, GPR68 (50), which was an additional candidate EET receptor in our bioinformatic analysis. Heterodimerization with GPR68 or other GPCRs could affect GPR132's acid responsiveness or its affinity for different fatty acids.

GPR132 is expressed in macrophages, and its previously described physiological roles include regulating macrophage activation $(29,51)$ and, in aging mice, the production of lymphoid cells (26). We have described for the first time a role for GPR132 in regulating HSPCs. This regulation could function autonomously in HSPCs or could occur via macrophages, which are present in developmental and adult HSPC niches and are known to regulate HSPC function (reviewed in ref. 52).

Beginning with bioinformatic analysis to deorphan the bioactive 11,12-EET, we uncovered an oxy-fatty acid-GPR132 signaling axis that regulates developmental and adult hematopoiesis. These results add to prior studies by our group demonstrating a role for prostaglandins in regulating HSPC development and transplant (53), as well as earlier work exploring the roles of lipid mediators such as HETEs, HODEs, and leukotrienes in regulating inflammation (54-57), B-cell production, adhesion, and $\mathrm{Ig}$ production (58-61) and T-cell function (62-64). Together, these reports show that complex networks of lipid-derived signaling molecules regulate diverse biological phenomena, including hematopoiesis and stem cell dynamics (65). GPR132 may represent a node in this network able to integrate the input of diverse lipidderived signals into a single prohematopoietic output. Systematic understanding of these networks is critically needed.

\section{Methods}

Materials. All fatty acids, EET-methyl ester, and isoproterenol were purchased from Cayman Chemicals at 5-10 mM stock concentrations in ethanol or DMSO. All chiral molecules were purchased as racemic mixtures. Histamine dihydrochloride (H7250), LPC (L1881), SPC (S4257) and lactic acid (L6661) were from Sigma-Aldrich and diluted in ethanol. Recombinant human chemerin and MIP-3 $\beta$ were from PeproTech, Inc.

PathHunter $\boldsymbol{\beta}$-Arrestin Assays. PathHunter $\beta$-arrestin assays were purchased from DiscoverX and performed in 384-well plates according to manufacturer instructions. Each small molecule was tested in a minimum of two separate experiments. $\mathrm{EC}_{50}$ values and nonlinear regressions were performed in GraphPad Prism.

Zebrafish Embryo Experiments. Zebrafish were housed and cared for according to Institutional Animal Care and Use Committee (IACUC) protocol 14-10-2789 with IRB approval from Boston Children's Hospital. Casper or AB zebrafish were used for all experiments. For MO injections, embryos were injected at the 1-cell stage with 4-6 ng MO, mixed with phenol red as an indicator of injection success. The Gpr132b_sp MO (sequence: TAAAATGGCGTTGCTCTTACCTCTA) and Standard Control MO (sequence: CCTCTTACCTCAGTTACAATTTATA) were produced by GeneTools. For small molecule treatments, embryos were dechorionated at $24 \mathrm{hpf}$, and small molecule was added to E3 media and embryos in 12-well plates. Embryos were fixed at 36 hpf in 4\% PFA.

Mouse Transplant. Mice were housed and cared for according to IACUC protocol 15-06-2964 with IRB approval from Boston Children's Hospital, or according to IACUC protocol 15-03-237 with IRB approval from Harvard University. GPR132 knockout mice (Jackson Labs \#008576) were kindly provided by Donna Bratton, University of Colorado, Denver. Mice were genotyped for GPR132 status by PCR, as previously described (26). The indicated number of donor cells were mixed with 100,000 CD45.2 wild-type (Jackson Labs \#000664), sex- and age-matched competitor marrow cells. Cells were transplanted retro-orbitally into sex- and age-matched CD45.2 wild-type recipients, which had received $10 \mathrm{gy}$ irradiation in a split dose. At 6 mo posttransplant, recipient mice were euthanized, and their marrow was analyzed for CD45.1 chimerism. Bone marrow from select representative mice was used in secondary transplants, and each secondary recipient received 2 million cells from a single primary recipient.

Antibody Staining and Flow Cytometry Analysis. Mouse peripheral blood was incubated with ACK lysing buffer (Thermo Fisher) to deplete red blood cells. Cells were incubated for $1 \mathrm{~h}$ at $4{ }^{\circ} \mathrm{C}$ with $1: 100$ dilutions of the following antibodies: Ter119-PE-Cy5 (red blood cells; eBioscience), CD3-APC (T cells; eBioscience), B220-Pacific Blue (B cells; eBioscience), Gr1-PE-Cy7 (clone RB6$8 C 5$, granulocytes; eBioscience), CD45.1-PE (BD Pharmingen), and CD45.2 FITC (BD Pharmingen). Cells were analyzed on an LSRIl Flow Cytometer. FlowJo software was used to process the data.

ACKNOWLEDGMENTS. This work was supported by NIH Grants R01HL04880 P01HL032262, U01HL100001, R24DK092760, and U01HL134812 (to L.I.Z.), 5F31HL129517-02 (to J.L.L.), and P01GM095467 (to C.N.S.). 
1. Samuelsson B, Dahlén SE, Lindgren JA, Rouzer CA, Serhan CN (1987) Leukotrienes and lipoxins: Structures, biosynthesis, and biological effects. Science 237:1171-1176.

2. Bellien J, Joannides R (2013) Epoxyeicosatrienoic acid pathway in human health and diseases. J Cardiovasc Pharmacol 61:188-196

3. Spector AA, Kim HY (2015) Cytochrome P450 epoxygenase pathway of poly unsaturated fatty acid metabolism. Biochim Biophys Acta 1851:356-365.

4. Panigrahy D, et al. (2012) Epoxyeicosanoids stimulate multiorgan metastasis and tumor dormancy escape in mice. J Clin Invest 122:178-191.

5. Li P, et al. (2015) Epoxyeicosatrienoic acids enhance embryonic haematopoiesis and adult marrow engraftment. Nature 523:468-471.

6. Baron A, Frieden M, Bény JL (1997) Epoxyeicosatrienoic acids activate a highconductance, $\mathrm{Ca}(2+)$-dependent $\mathrm{K}+$ channel on pig coronary artery endothelia cells. J Physiol 504:537-543.

7. Zhang $Y$, et al. (2001) EET homologs potently dilate coronary microvessels and activate BK(Ca) channels. Am J Physiol Heart Circ Physiol 280:H2430-H2440.

8. Snyder GD, Krishna UM, Falck JR, Spector AA (2002) Evidence for a membrane site of action for 14,15-EET on expression of aromatase in vascular smooth muscle. $\mathrm{Am}$ Physiol Heart Circ Physiol 283:H1936-H1942.

9. Chen Y, Falck JR, Manthati VL, Jat JL, Campbell WB (2011) 20-lodo-14,15-epoxyeicosa8(Z)-enoyl-3-azidophenylsulfonamide: Photoaffinity labeling of a 14,15-epoxyeicosatrienoic acid receptor. Biochemistry 50:3840-3848.

10. Li PL, Campbell WB (1997) Epoxyeicosatrienoic acids activate K+ channels in coronary smooth muscle through a guanine nucleotide binding protein. Circ Res 80:877-884.

11. Ding $Y$, et al. (2014) The biological actions of 11,12-epoxyeicosatrienoic acid in endothelial cells are specific to the $\mathrm{R} / \mathrm{S}$-enantiomer and require the $\mathrm{G}(\mathrm{s})$ protein J Pharmacol Exp Ther 350:14-21.

12. Yang $W$, et al. (2008) Characterization of epoxyeicosatrienoic acid binding site in U937 membranes using a novel radiolabeled agonist, 20-125i-14,15-epoxyeicosa-8(Z) enoic acid. J Pharmacol Exp Ther 324:1019-1027.

13. Yin $\mathrm{H}$, et al. (2009) Lipid $\mathrm{G}$ protein-coupled receptor ligand identification using betaarrestin PathHunter assay. J Biol Chem 284:12328-12338.

14. Rolin J, Al-Jaderi Z, Maghazachi AA (2013) Oxidized lipids and lysophosphatidylcholine induce the chemotaxis and intracellular calcium influx in natural killer cells. Immunobiology 218:875-883.

15. Obinata H, Hattori T, Nakane S, Tatei K, Izumi T (2005) Identification of 9-hydroxyoctadecadienoic acid and other oxidized free fatty acids as ligands of the $\mathrm{G}$ proteincoupled receptor G2A. J Biol Chem 280:40676-40683.

16. Hohmann SW, et al. (2017) The G2A receptor (GPR132) contributes to oxaliplatininduced mechanical pain hypersensitivity. Sci Rep 7:446.

17. Wong PY, Lai PS, Shen SY, Belosludtsev YY, Falck JR (1997) Post-receptor signa transduction and regulation of 14(R),15(S)-epoxyeicosatrienoic acid (14,15-EET) binding in U-937 cells. J Lipid Mediat Cell Signal 16:155-169.

18. Fleming I (2007) Epoxyeicosatrienoic acids, cell signaling and angiogenesis. Prostaglandins Other Lipid Mediat 82:60-67.

19. Krishnamoorthy $S$, et al. (2010) Resolvin D1 binds human phagocytes with evidence for proresolving receptors. Proc Natl Acad Sci USA 107:1660-1665.

20. Liu $X$, et al. (2016) Functional screening for $G$ protein-coupled receptor targets of 14,15-epoxyeicosatrienoic acid. Prostaglandins Other Lipid Mediat 132:31-40.

21. Murakami N, Yokomizo T, Okuno T, Shimizu T (2004) G2A is a proton-sensing G protein-coupled receptor antagonized by lysophosphatidylcholine. J Biol Chem 279 42484-42491.

22. Ichijo $Y$, et al. (2016) Two zebrafish G2A homologs activate multiple intracellular signaling pathways in acidic environment. Biochem Biophys Res Commun 469:81-86.

23. Ludwig MG, et al. (2003) Proton-sensing G-protein-coupled receptors. Nature 425 : 93-98.

24. Radu CG, Nijagal A, McLaughlin J, Wang L, Witte ON (2005) Differential proton sensitivity of related $G$ protein-coupled receptors $T$ cell death-associated gene 8 and G2A expressed in immune cells. Proc Natl Acad Sci USA 102:1632-1637.

25. Xu J, Wang T, Wu Y, Jin W, Wen Z (2016) Microglia colonization of developing zebrafish midbrain is promoted by apoptotic neuron and lysophosphatidylcholine. Dev Cell 38:214-222.

26. Le LQ, et al. (2001) Mice lacking the orphan G protein-coupled receptor G2A develop a late-onset autoimmune syndrome. Immunity 14:561-571.

27. Liu JY, et al. (2013) Substituted phenyl groups improve the pharmacokinetic profile and anti-inflammatory effect of urea-based soluble epoxide hydrolase inhibitors in murine models. Eur J Pharm Sci 48:619-627.

28. Frömel T, et al. (2012) Soluble epoxide hydrolase regulates hematopoietic progenitor cell function via generation of fatty acid diols. Proc Natl Acad Sci USA 109:9995-10000.

29. Chen $P$, et al. (2017) Gpr132 sensing of lactate mediates tumor-macrophage interplay to promote breast cancer metastasis. Proc Natl Acad Sci USA 114:580-585.

30. Kabarowski JH (2009) G2A and LPC: Regulatory functions in immunity. Prostaglandins Other Lipid Mediat 89:73-81.

31. Kabarowski JH, Zhu K, Le LQ, Witte ON, Xu Y (2001) Lysophosphatidylcholine as a ligand for the immunoregulatory receptor G2A. Science 293:702-705.

32. Witte ON, Kabarowski JH, Xu Y, Le LQ, Zhu K (2005) Retraction. Science 307:206.

33. Loot AE, et al. (2012) 11,12-EET stimulates the association of BK channel $\alpha$ and $\beta(1)$ subunits in mitochondria to induce pulmonary vasoconstriction. PLoS One 7:e46065.

34. Yan G, Chen S, You B, Sun J (2008) Activation of sphingosine kinase-1 mediates induction of endothelial cell proliferation and angiogenesis by epoxyeicosatrienoic acids. Cardiovasc Res 78:308-314.
35. Zeldin DC, et al. (1993) Regio- and enantiofacial selectivity of epoxyeicosatrienoic acid hydration by cytosolic epoxide hydrolase. J Biol Chem 268:6402-6407.

36. Rand AA, et al. (2017) Cyclooxygenase-derived proangiogenic metabolites of epoxyeicosatrienoic acids. Proc Natl Acad Sci USA 114:4370-4375.

37. Kacik M, Oliván-Viguera A, Köhler R (2014) Modulation of $\mathrm{K}(\mathrm{Ca}) 3.1$ channels by eicosanoids, omega-3 fatty acids, and molecular determinants. PLoS One 9:e112081.

38. Park SK, et al. (2018) GPR40 is a low-affinity epoxyeicosatrienoic acid receptor in vascular cells. J Bio/ Chem 293:10675-10691.

39. Pascual JM, McKenzie A, Yankaskas JR, Falck JR, Zeldin DC (1998) Epoxygenase metabolites of arachidonic acid affect electrophysiologic properties of rat tracheal epithelial cells1. J Pharmacol Exp Ther 286:772-779.

40. Spector AA, John K, Fletcher JE (1969) Binding of long-chain fatty acids to bovine serum albumin. J Lipid Res 10:56-67.

41. Ma Y, et al. (2015) Epoxyeicosatrienoic acids act through TRPV4-TRPC1-KCa1.1 complex to induce smooth muscle membrane hyperpolarization and relaxation in human internal mammary arteries. Biochim Biophys Acta 1852:552-559.

42. Fleming l, et al. (2007) Epoxyeicosatrienoic acids regulate Trp channel dependent $\mathrm{Ca}+$ signaling and hyperpolarization in endothelial cells. Arterioscler Thromb Vasc Biol 27:2612-2618.

43. Watanabe $\mathrm{H}$, et al. (2003) Anandamide and arachidonic acid use epoxyeicosatrienoic acids to activate TRPV4 channels. Nature 424:434-438.

44. Wray JA, et al. (2009) The epoxygenases CYP2J2 activates the nuclear receptor PPARalpha in vitro and in vivo. PLoS One 4:e7421

45. Yang C, et al. (2010) 14,15-Epoxyeicosatrienoic acid induces vasorelaxation through the prostaglandin EP(2) receptors in rat mesenteric artery. Prostaglandins Other Lipid Mediat 93:44-51.

46. Ma SK, et al. (2015) Overexpression of G-protein-coupled receptor 40 enhances the mitogenic response to epoxyeicosatrienoic acids. PLoS One 10:e0113130.

47. Patwardhan AM, Scotland PE, Akopian AN, Hargreaves KM (2009) Activation of TRPV1 in the spinal cord by oxidized linoleic acid metabolites contributes to inflammatory hyperalgesia. Proc Natl Acad Sci USA 106:18820-18824.

48. Seuwen K, Ludwig MG, Wolf RM (2006) Receptors for protons or lipid messengers or both? J Recept Signal Transduct Res 26:599-610.

49. Tomura H, Mogi C, Sato K, Okajima F (2005) Proton-sensing and lysolipid-sensitive Gprotein-coupled receptors: A novel type of multi-functional receptors. Cell Signal 17: 1466-1476.

50. Huang YH, Su YS, Chang CJ, Sun WH (2016) Heteromerization of G2A and OGR1 enhances proton sensitivity and proton-induced calcium signals. I Recept Signal Transduct Res 36:633-644.

51. Bolick DT, et al. (2009) G2A deficiency in mice promotes macrophage activation and atherosclerosis. Circ Res 104:318-327.

52. McCabe A, MacNamara KC (2016) Macrophages: Key regulators of steady-state and demand-adapted hematopoiesis. Exp Hematol 44:213-222.

53. North TE, et al. (2007) Prostaglandin E2 regulates vertebrate haematopoietic stem cell homeostasis. Nature 447:1007-1011.

54. Powell WS, Rokach J (2015) Biosynthesis, biological effects, and receptors of hydroxyeicosatetraenoic acids (HETEs) and oxoeicosatetraenoic acids (oxo-ETEs) derived from arachidonic acid. Biochim Biophys Acta 1851:340-355.

55. Shapiro $H$, Singer P, Ariel A (2016) Beyond the classic eicosanoids: Peripherallyacting oxygenated metabolites of polyunsaturated fatty acids mediate pain associated with tissue injury and inflammation. Prostaglandins Leukot Essent Fatty Acids 111:45-61

56. Bäck M (2007) Leukotriene receptors: Crucial components in vascular inflammation. Sci World J 7:1422-1439.

57. Massoumi R, Sjölander A (2007) The role of leukotriene receptor signaling in inflammation and cancer. Sci World J 7:1413-1421.

58. Lauder SN, et al. (2017) Myeloid 12/15-LOX regulates B cell numbers and innate immune antibody levels in vivo. Wellcome Open Res 2:1.

59. Liu X, Zhu P, Freedman BD (2006) Multiple eicosanoid-activated nonselective cation channels regulate B-lymphocyte adhesion to integrin ligands. Am J Physiol Cell Physiol 290:C873-C882.

60. Lamoureux J, Stankova J, Rola-Pleszczynski M (2006) Leukotriene D4 enhances immunoglobulin production in CD40-activated human B lymphocytes. J Allergy Clin Immunol 117:924-930.

61. Yamaoka KA, Claésson HE, Rosén A (1989) Leukotriene B4 enhances activation, proliferation, and differentiation of human B lymphocytes. J Immunol 143:1996-2000.

62. Yang XY, et al. (2002) Interleukin (IL)-4 indirectly suppresses IL-2 production by human $\mathrm{T}$ lymphocytes via peroxisome proliferator-activated receptor gamma activated by macrophage-derived 12/15-lipoxygenase ligands. J Biol Chem 277:3973-3978.

63. Luster AD, Tager AM (2004) T-cell trafficking in asthma: Lipid mediators grease the way. Nat Rev Immunol 4:711-724.

64. Goodwin JS (1986) Regulation of T cell activation by leukotriene B4. Immunol Res 5: 233-248.

65. Tsoyi K, et al. (2016) Carbon monoxide improves efficacy of mesenchymal stromal cells during sepsis by production of specialized proresolving lipid mediators. Crit Care Med 44:e1236-e1245. 